\title{
Pregnancy with Atypical Pulmonary Tuberculosis During the COVID-19 Outbreak: A Case Report
}

\author{
Ai-Ping ZHANG ( $18895338133 @ 163 . c o m$ ) \\ Yijishan Hospital of Wannan Medical College \\ Manman Liang \\ Yijishan Hospital of Wannan Medical College

\section{Zijian Wang} \\ Yijishan Hospital of Wannan Medical College

\section{Weishun Hou} \\ Yijishan Hospital of Wannan Medical College \\ Jianghua Yang \\ Yijishan Hospital of Wannan Medical College
}

\section{Case Report}

Keywords: Tuberculosis, Pregnancy, Case report

Posted Date: May 11th, 2021

DOI: https://doi.org/10.21203/rs.3.rs-487294/v1

License: (c) (i) This work is licensed under a Creative Commons Attribution 4.0 International License. Read Full License 


\section{Abstract}

Background: During pregnancy, due to reduced immunological resistance, the probability of tuberculosis infection is increased, easily leading to the occurrence of tuberculosis; thus, pregnancy with tuberculosis is a risk to the health of pregnant women with infectious diseases. However, the clinical symptoms of pregnancy with pulmonary tuberculosis are atypical, often confused with the physiological reactions of pregnancy, and there is a lack of clinical awareness, especially in the context of the global COVID-19 pandemic. Specifically, atypical lung lesions are prone to misdiagnosis or delayed diagnosis.

Case presentation: A 21-year-old woman was infected with Mycobacterium tuberculosis during the third trimester of pregnancy. The diagnosis and treatment of the patient are reported.

Conclusion: The interaction between tuberculosis and pregnancy is a double-edged sword. Increasing awareness of tuberculosis among doctors is essential, and early diagnosis and standardized treatment are key to improving the outcome of pregnancy.

\section{Background}

Tuberculosis (TB) is one of the most important infectious diseases and endangers all humankind. Nearly one-fourth of the world population has TB infection[1]. Statistically, TB is one of the top three causes of death among women aged 15-45 years[2]. Pregnancy with TB is defined as the development of TB during pregnancy, the presence of TB infection in a woman of childbearing age who becomes pregnant, or a diagnosis of TB within three months after delivery. It is estimated that more than three million women worldwide are infected with TB and are in the incubation period[3]. Approximately 700,000 women die of TB each year, and approximately $26 \%$ of all preventable deaths in pregnancy globally are directly attributable to TB[4]. In China, pregnant patients with TB account for $2 \% \sim 7 \%$ of the all pregnancies[5]. In 2016, Sobhy. S summarized relevant studies on the outcomes of pregnant women with active TB during pregnancy and proposed that active TB during pregnancy was associated with adverse outcomes for both the mother and the foetus[6]. Because of the global COVID-19 pandemic, pulmonary diseases have attracted increasing attention. This article reviews the diagnosis and treatment of a case of pregnancy with atypical TB and the relevant literature to provide a reference for the diagnosis of TB in pregnancy in the future.

\section{Case Presentation}

\section{General information and epidemiological history}

A 21-year-old woman, weighing $55 \mathrm{~kg}$ and measuring $160 \mathrm{~mm}$ in height, who freelanced, was in her first pregnancy, at her 28th gestational week. The patient denied having TB, but her husband had the disease and was cured one year ago. The patient had no history of hypertension or diabetes and denied other infectious diseases or chronic diseases. The patient lived in Wuhu for a long time, denied leaving Wuhu for nearly one month, and denied having contact with COVID-19 patients. 


\section{Symptoms and signs on admission}

The patient developed cough, sputum and low fever on January 3, 2021. Routine blood examination on January 5 showed that her leukocyte count was $7.5^{\star} 10^{\wedge} 9 / \mathrm{L}$, percentage of mesenchytes was $85.8 \%$, percentage of lymphocytes was $9.5 \%$, neutrophil count was $6.4^{\star} 10^{\wedge} 9 / \mathrm{L}$, and lymphocyte count was $0.7 * 10^{\wedge} 9 / \mathrm{L}$. Abdominal B-mode ultrasonography showed spleen enlargement and no obvious abnormalities in the liver, gallbladder, pancreas or kidneys. Cardiac colour ultrasonography showed abnormal filling of the left ventricle (considered to be caused by tachycardia) and tachycardia (real-time heart rate: $128 \mathrm{bpm}$ ). The patient refused a chest imaging examination because of her pregnancy status. The initial diagnosis was "upper respiratory tract infection, bronchitis?".

The patients were given Lianhua Qingwen granules for detoxification and anti-infection treatment with cefclo for 5 days. Afterwards, the patient's fever broke, but her cough did not improve. On January 10, the patient developed dyspnoea with progressive aggravation and was admitted to Yijishan Hospital of Wannan Medical College on January 13. Physical examination on admission showed the following: T, $36.9^{\circ} \mathrm{C} ; \mathrm{P}, 145$ times/min; R, 27 times/min; BP, 124/97 mmHg; and SpO2 (73\%). Wet rales could be heard in both lungs. The rhythm was uniform, and no abnormal heart sounds were heard.

\section{Diagnosis and treatment process}

The patient was admitted to the intensive care unit (ICU) unit due to dyspnoea, and pulse oximetry of the finger showed decreased oxygenation to approximately $75 \%$. After hospitalization, the patient was given non-invasive ventilator-assisted breathing. On January 13th, blood cell analysis showed the following: leukocyte count, $8.5^{\star} 10^{\wedge} 9 / \mathrm{L}$; neutrophil percentage, $90.7 \%$; lymphoid cell percentage, $7.5 \%$; neutrophil count, $7.7 * 10^{\wedge} 9 / \mathrm{L}$; lymphocyte count, $0.6 * 10^{\wedge} 9 / \mathrm{L}$ : hypersensitive C-reactive protein, $164.76 \mathrm{mg} / \mathrm{L}$; and procalcitonin, 1.028 ng/L. Her 2019-nCoV nucleic acid, H1N1 nucleic acid, and HIV antibody tests were negative. After the adverse effects of radiation and drugs on the foetus were explained, the family members were fully informed of the patient's condition, and the informed consent form was signed. Then, chest CT was completed to show the infective lesions of both lungs (Fig.1). The initial diagnosis was "acute respiratory failure and severe pneumonia at 28 weeks of gestation". Because the pathogens causing the pulmonary inflammation were unknown, an antiviral (oral oseltamivir $75 \mathrm{mg}$ bid), antiinfection treatment (linezolid glucose injection $0.6 \mathrm{~g} \mathrm{q} 12 \mathrm{~h}$ combined with imipenem CILAStatin $1 \mathrm{~g} \mathrm{q} 8 \mathrm{~h}$ intravenous drip), and maintenance of water and electrolyte balance were given. After two hours of noninvasive ventilator oxygen therapy, the patient's hypoxemia could not be corrected, and her partial oxygen pressure of arterial blood gas was $67.4 \mathrm{mmHg}$. Afterward, the patient was given invasive ventilatorassisted breathing. After pulse oximetry of the patient's finger was performed, her blood pressure and heart rhythm appeared stable, and bedside fibreoptic bronchoscopy and bronchoalveolar lavage were performed. During the operation, the right main bronchus and lower lobe bronchus were unobstructed, and the mucous membrane was slightly congested without obvious sputum; the lumen of the left main bronchus and lower lobe bronchus was unobstructed, the mucosa was slightly congested, and 
transparent exudate could be seen and sucked out under negative pressure. Bronchoalveolar lavage fluid was obtained for pathogen examination.

On January 16 , the culture results were negative for bacteria and fungi in the lavage fluid. On January 18 , a sputum smear was positive for acid-fast bacilli, a TB infection T cell test was positive, and there were antigen A holes (119) and antigen B holes (92). After 5 days of treatment, the chest tightness of the patient was improved, and her vital signs were stable. On January 18 , the ventilator was successfully withdrawn and changed to high-flow oxygen inhalation. On January 19th, the patient was transferred to the TB ward and treated with isoniazid (INH), rifampicin (RFP), ethambutol (ETB) and pyrazinamide (PRZ). The clinical symptoms of the patient gradually improved, the foetus developed well, and there were no abnormalities after monitoring. On January 27th, re-examination by chest CT showed that the infectious lesions of both lungs were absorbed. (Fig. 2). The patient was discharged with medicine on January 29. On March 10, her culture results were positive for Mycobacterium tuberculosis and indicated sensitivity to first-line anti-TB drugs.

\section{Discussion}

In China, women of childbearing age receive active attention as a population with a high incidence of TB. TB is also the leading cause of death among women worldwide. According to statistics, more than one million women die every year, among which active TB is the main cause of maternal mortality[7]. Therefore, it is necessary to raise awareness of the risk of TB in pregnancy.

The causes of TB in pregnancy may be as follows. First, anti-TB drugs lead to a decrease in the efficacy of contraceptives. The conventional anti-TB drug rifampicin, when used in combination with contraceptives (levonorgestrel), can increase the metabolism of steroid contraceptives and increase the plasma clearance rates of ethinylestradiol and norethisterone[8], resulting in accidental pregnancy during anti-TB treatment and increasing the incidence of pregnancy with TB[9]. Second, the cellular immune function of the body decreases[2]. The high level of human chorionic gonadotropin in pregnancy can inhibit the immune function of lymphocytes, and the early pregnancy response affects the nutritional absorption of pregnant women, resulting in metabolic disorder and then leading to a decrease in cellular immune function, which is not conducive to the clearance of TB. Third, the change in endocrine hormone levels is affected by increased ovarian hormones during pregnancy, which leads to disorders of autonomic nervous regulation, endocrine and metabolic function, lung hyperaemia, and upper respiratory tract swelling, among others. Adrenocortical hormone secretion in pregnant women is high, and capillary permeability increases, resulting in susceptibility to infection by Mycobacterium tuberculosis or recurrence of the original TB focus[10]. Fourth, in pregnant women, with the increase in circulating blood volume, microvascular permeability increases gradually, and the upper respiratory tract becomes congested and swollen, which is very beneficial for TB bacterial invasion into blood circulation. Pregnancy causes hypoxia in the lungs due to the rise of the diaphragm and decrease in lung dilatation, resulting in lung susceptibility to TB infection[11]. Fifth, postpartum TB infection or recurrence may be 
caused by rapid changes in hormone levels, changes in cellular immunity, the lowering of the diaphragm, nutritional consumption and a lack of sleep[12].

TB is an independent risk factor during pregnancy. Delayed diagnosis remains associated with a substantially elevated risk for poor maternal and foetal outcomes, including a threefold increase in maternal morbidity, ninefold increase in miscarriage, twofold increase in preterm birth and low birthweight, and sixfold increase in perinatal death $[6,13]$. Therefore, the key to pregnancy with pulmonary TB is early diagnosis. However, TB during pregnancy is not easily diagnosed because the general symptoms after TB infection are nonspecific and manifest mainly as respiratory symptoms and signs: cough, expectoration, haemoptysis, low fever, night sweats, chest pain, shortness of breath and emaciation[7, 14]. It should be mentioned that the symptoms of TB are easily confused with the early pregnancy response. In the second or third trimester of pregnancy, if patients have cough, chest tightness, chest pain and related signs, it is difficult to distinguish them from pregnancy with pneumonia, so the diagnosis of TB is easily delayed in the clinic. The clinical presentation of this patient was similar to that of patients with the common symptoms of novel coronavirus infection (fever, cough, and dyspnoea), and this case was extremely difficult to differentiate from a typical COVID-19 case on the basis of clinical presentation alone. Therefore, to ensure the health of pregnant women and new-borns, it is urgent to make accurate diagnoses as soon as possible for patients with suspected pregnancy with TB[15]. Currently, the diagnosis of TB is based mainly on its epidemiology, clinical manifestations, imaging, bacterial pathology and immunology. $\otimes$ Aetiological examination (sputum smear or culture, TB antigen, antibody detection, etc.) is non-invasive, easily accepted by the patient, and can be used as the first choice approach during pregnancy; however, the sensitivity of acid-fast bacilli staining is low, so more than three sputum examinations are recommended to avoid a missed diagnosis. The sensitivity and specificity of isolation and culture of TB bacteria are relatively high, so it is recommended that pregnant women undergo isolation and culture evaluations for TB bacteria, and sputum culture can be used as the gold standard for diagnosis. $\triangle$ Blood biochemical and immunological tests (routine blood tests, the erythrocyte sedimentation rate, the cutaneous tuberculin test (TST), TB T cell dot test (T-SPOT.TB), etc.) and the TST have been proven to be safe and effective during pregnancy, and the experimental results are not affected by pregnancy. Chen et al.[16] found that T-SPOT.TB is highly sensitive for detection of active TB during pregnancy. It is suggested that interferon gamma release assays (IGRAs) be used in clinical practice to screen for TB in pregnancy. IGRAs during pregnancy may be more specific and sensitive than the TST, and the experimental results are not significantly related to pregnancy[17], thereby providing a more accurate method for the diagnosis of TB in pregnancy. $\otimes$ Chest imaging examination (chest $\mathrm{X}$-ray examination, $\mathrm{CT}$ examination and magnetic resonance imaging (MRI) examination, etc.) is also an option, but imaging examinations of pregnant women are rarely performed clinically because of concerns about the effects of radiation on foetal growth and development. Therefore, imaging examination is used only when pulmonary TB is highly suspected and requires the informed consent of the patient. The diagnostic value of CT is better than that of chest X-ray, while MRI is better as an alternative examination method in the early stage of pregnancy. The 2004 guidelines for diagnostic imaging during pregnancy from the American College of Obstetricians and Gynecologists (ACOG) state 
that the dose of radiation in diagnostic tests is not sufficient to affect the developing embryo or foetus. If the dose of X-rays during pregnancy is less than $5 \mathrm{rad}$, there is no effect on foetal development. In fact, the exposure dose of diagnostic radioactivity without contrast agent is less than 5 rad. The 2016 ACOG guidelines also state that because the amount of radiation used in X-rays, CT scans and nuclear medicine imaging is far lower than the dose that would cause harm to the foetus, there is no need to withhold this option from pregnant patients if it is clinically necessary or if diagnostic problems can be more easily addressed. The latest guidelines for imaging tests during pregnancy and lactation indicate that imaging tests such as X-rays and CT are safe during pregnancy and lactation and that the radiation dose is far lower than the ionizing radiation dose that can cause damage to the foetus[18]. However, embryos at 8 to 15 weeks of gestation are most sensitive to radiation, and the effect is non-dose-dependent[19]. Therefore, it is necessary to comprehensively consider the pregnant woman's condition and gestational week and decide whether to carry out radiological examination after weighing the advantages and disadvantages. The laboratory examination of this patient showed that the infection index increased and the lymphocyte count decreased, which was consistent with the changes associated with SARS-CoV-2 infection in laboratory examinations. SARS-CoV-2 nucleic acid testing of pharyngeal swabs was performed in this patient, and the results were negative. Because the nucleic acid test had a certain false negative rate, serum SARS-CoV-2-specific $\operatorname{lgM} / \operatorname{lgG}$ antibody was measured, and the result was negative; thus, SARS-CoV-2 infection was excluded. Although chest CT showed no COVID-19 multiple ground-glass opacity in either lung, viral pneumonia could not be ruled out. Although the patient had a history of TB exposure, the diagnostic basis of TB was insufficient combined with chest CT. Therefore, sputum samples of the patient were obtained to detect Mycobacterium tuberculosis, the test results were positive, and the results of the T-SPOT.TB test were positive, which confirmed TB infection.

For pregnancy with pulmonary TB, there is a need not only for early diagnosis but also to grasp the opportunity for treatment. It is generally believed that TB with standard treatment does not have adverse effects on the foetus[20]. Delayed diagnosis or nonstandard treatment can lead to an increased risk of abortion, intrauterine infection, foetal death and intrauterine and neonatal mortality. Jana et al.[20] reported that perinatal mortality increased by 5 fold among 79 cases of pregnancy with active TB in India. Figueroa et al.[21] reported that TB infection during pregnancy increased the morbidity and mortality of new-borns, and that the later the start of anti-TB treatment was, the more obvious the results were. Given poor maternal and foetal outcomes with untreated active TB disease, the benefits of treatment outweigh the potential risks from medications[6]. In terms of drugs, a growing number of reports have shown that first-line anti-TB drugs, including rifampicin, isoniazid, pyrazinamide and ethambutol, have no adverse effects on foetuses at conventional doses[22, 23]. All first-line drugs, except for streptomycin, which has toxic effects on the foetus, can be used during pregnancy[24]. The patient was in the second or third trimester of pregnancy and showed no specific discomfort after anti-TB treatment with INH, RFP, EMB and PZA. There was no apparent foetal abnormality after re-examination, so labour induction was not performed. Lesion absorption improved, and the patient was discharged from the hospital. Most scholars believe that TB is not an indication for termination of pregnancy but that termination of pregnancy should be recommended under the following circumstances: (1) Active 
pulmonary TB in early pregnancy that needs timely anti-TB treatment, while considering the unavoidable adverse effects of drugs on the foetus; (2) Severe pulmonary TB with decreased lung function, where the patient is unable to tolerate continued pregnancy and delivery; (3) AIDS patients who develop TB during pregnancy; and (4) Pregnancy with other systemic diseases that cannot be continued.

\section{Conclusions}

In short, the interaction between TB and pregnancy is a double-edged sword. On the one hand, pregnancy affects the progress of TB, and on the other hand, TB affects pregnancy and the new-born. Early detection, early diagnosis and early treatment are key to determining prognosis. For patients with atypical clinical symptoms in the context of the COVID-19 pandemic, it is recommended to conduct chest imaging examinations and follow the principle of the lowest dose as much as possible. There is no obvious harm to the foetus caused by the use of X-rays and CT during pregnancy. At the same time, increased awareness of TB among doctors is essential, and early diagnosis and standardized treatment are key to improving the outcome of pregnancy.

\section{Declarations}

\section{Acknowledgments}

Not applicable.

\section{Authors' contributions}

$\mathrm{ZP}$ and $\mathrm{JH}$ designed the study. MM and ZJ analyzed the data and wrote the initial draft of the manuscript. HS collected the patients data and analyzed the data. All authors read and approved the final manuscript.

\section{Funding}

There was no funding from any source for this case report.

\section{Availability of data and materials}

All the relevant data is included in the case report. Reasonable request for any additional data can contact with the corresponding author.

\section{Ethics approval and consent to participate}

The present study was approved by The First Affiliated Yijishan Hospital of Wannan Medical College (Wuhu, China) and it conforms to the provisions of the Declaration of Helsinki. Written informed consent was obtained from all individuals in the present study. 
Written informed consent was obtained from the patient for publication of this case report and any accompanying figures or images. A copy of the written consent is available for review..

\section{Competing interests}

The authors have no conflicts of interest.

\section{References}

1. Miele K, Bamrah Morris S, Tepper NK. Tuberculosis in Pregnancy. Obstet Gynecol. 2020;135:144453.

2. Nguyen $\mathrm{H}$, Pandolfini $\mathrm{C}$, Chiodini P, Bonati M. Tuberculosis care for pregnant women: a systematic review. BMC Infect Dis. 2014;14:617.

3. van de Water B, Brooks M, Huang C, Trevisi L, Lecca L, Contreras C, et al. Tuberculosis clinical presentation and treatment outcomes in pregnancy: a prospective cohort study. BMC Infect Dis. 2020;20:686.

4. Castelo A, Mathiasi P, lunes R, Kritski A, Dalcolmo M, Fiuza de Melo F, et al. Cost effectiveness of antituberculosis interventions. PharmacoEconomics. 1995;8:385-99.

5. Mathad J, Gupta A. Tuberculosis in pregnant and postpartum women: epidemiology, management, and research gaps. Clinical infectious diseases: an official publication of the Infectious Diseases Society of America. 2012;55:1532-49.

6. Sobhy S, Babiker Z, Zamora J, Khan K, Kunst H. Maternal and perinatal mortality and morbidity associated with tuberculosis during pregnancy and the postpartum period: a systematic review and meta-analysis. BJOG: an international journal of obstetrics gynaecology. 2017;124:727-33.

7. Zumla A, Bates M, Mwaba P. The neglected global burden of tuberculosis in pregnancy. The Lancet Global health. 2014;2:e675-6.

8. Simmons K, Haddad L, Nanda K, Curtis K. Drug interactions between rifamycin antibiotics and hormonal contraception: a systematic review. BJOG: an international journal of obstetrics gynaecology. 2018;125:804-11.

9. Bothamley G, Ehlers C, Salonka I, Skrahina A, Orcau A, Codecasa L, et al. Pregnancy in patients with tuberculosis: a TBNET cross-sectional survey. BMC Pregnancy Childbirth. 2016;16:304.

10. Singh N, Perfect J. Immune reconstitution syndrome and exacerbation of infections after pregnancy. Clinical infectious diseases: an official publication of the Infectious Diseases Society of America. 2007;45:1192-9.

11. Leidecker K, Dorman K. Pulmonary Disorders Complicating Pregnancy: An Overview. J Perinat Neonatal Nurs. 2016;30:45-53.

12. Leung N, Bridoux F, Hutchison CA, Nasr SH, Cockwell P, Fermand JP, et al. Monoclonal gammopathy of renal significance: when MGUS is no longer undetermined or insignificant. Blood. 2012;120:42925. 
13. Sugarman J, Colvin C, Moran A, Oxlade O. Tuberculosis in pregnancy: an estimate of the global burden of disease. The Lancet Global health. 2014;2:e710-6.

14. Getahun H, Sculier D, Sismanidis C, Grzemska M, Raviglione M. Prevention, diagnosis, and treatment of tuberculosis in children and mothers: evidence for action for maternal, neonatal, and child health services. The Journal of infectious diseases 2012:S216-227.

15. Jana N, Barik S, Arora N. Re. Maternal and perinatal outcomes of tuberculosis during pregnancy and the postpartum period: a systematic review and meta-analysis: Maternal tuberculosis and undernutrition-an ominous combination for adverse maternal and perinatal outcomes. BJOG: an international journal of obstetrics and gynaecology 2017,124:2018-2019.

16. Chen Q, Guo X, Wang X, Wang M. T-SPOT.TB in Detection of Active Tuberculosis During Pregnancy: A Retrospective Study in China. Medical science monitor: international medical journal of experimental clinical research. 2016;22:57-60.

17. Malhamé I, Cormier M, Sugarman J, Schwartzman K. Latent Tuberculosis in Pregnancy: A Systematic Review. PloS one. 2016;11:e0154825.

18. Jain CACOG, Committee Opinion. Guidelines for Diagnostic Imaging During Pregnancy and Lactation. Obstetrics gynecology. 2019;No. 723:133:186.

19. Tirada N, Dreizin D, Khati N, Akin E, Zeman R. Imaging Pregnant and Lactating Patients. Radiographics: a review publication of the Radiological Society of North America Inc. 2015;35:175165.

20. Jana N, Barik S, Arora N, Singh A. Tuberculosis in pregnancy: the challenges for South Asian countries. J Obstet Gynaecol Res. 2012;38:1125-36.

21. Figueroa-Damián R, Arredondo-García J. Neonatal outcome of children born to women with tuberculosis. Arch Med Res. 2001;32:66-9.

22. Bothamley G. Drug treatment for tuberculosis during pregnancy: safety considerations. Drug safety. 2001;24:553-65.

23. Czeizel A, Rockenbauer M, Olsen J, Sørensen H. A population-based case-control study of the safety of oral anti-tuberculosis drug treatment during pregnancy. The international journal of tuberculosis lung disease: the official journal of the International Union against Tuberculosis Lung Disease. 2001;5:564-8.

24. Esmail A, Sabur N, Okpechi I, Dheda K. Management of drug-resistant tuberculosis in special subpopulations including those with HIV co-infection, pregnancy, diabetes, organ-specific dysfunction, and in the critically ill. Journal of thoracic disease. 2018;10:3102-18.

\section{Figures}



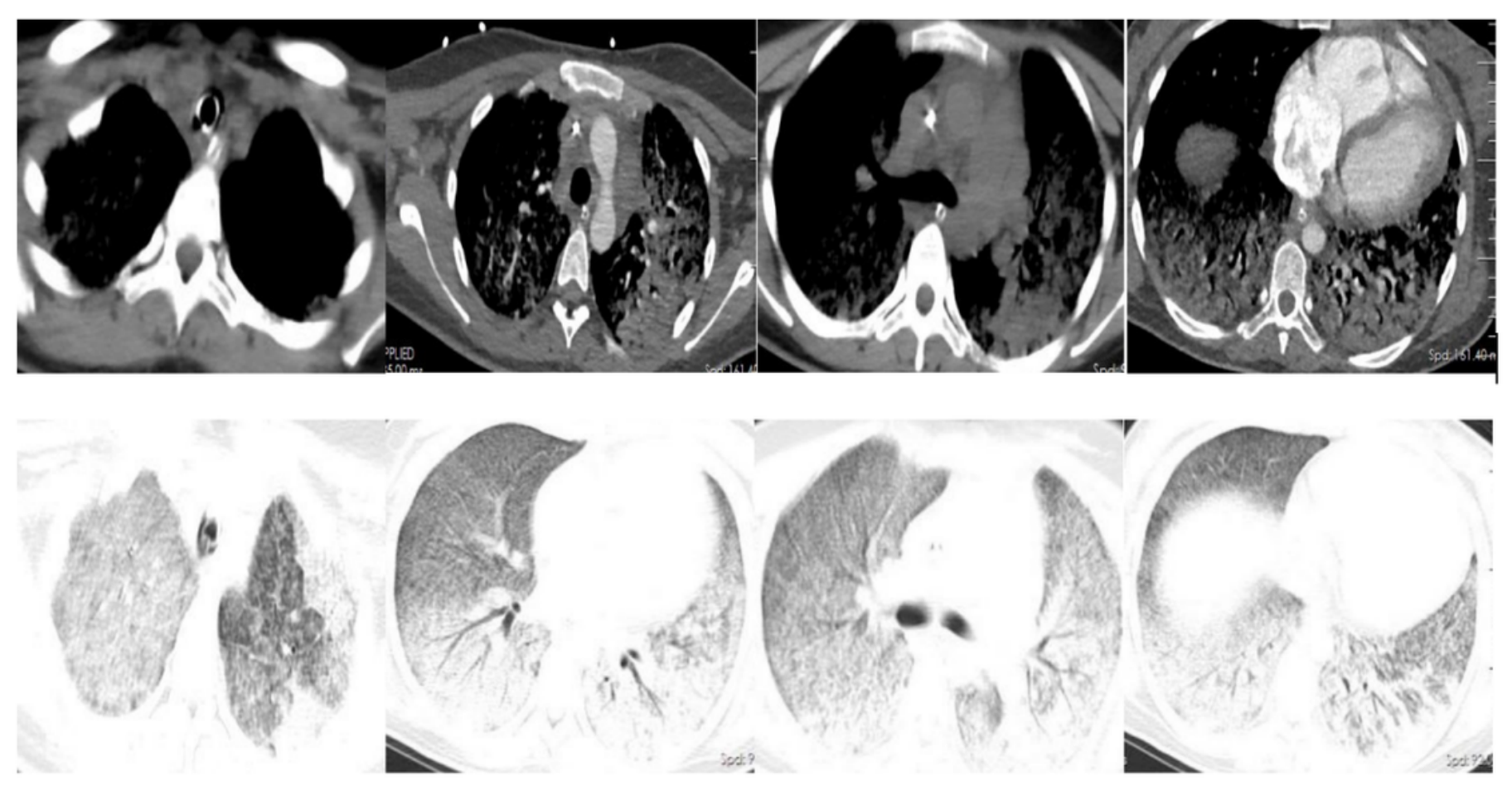

Graph 1

Figure 1

Chest CT shows infective lesion in both lungs(January 13, 2021).
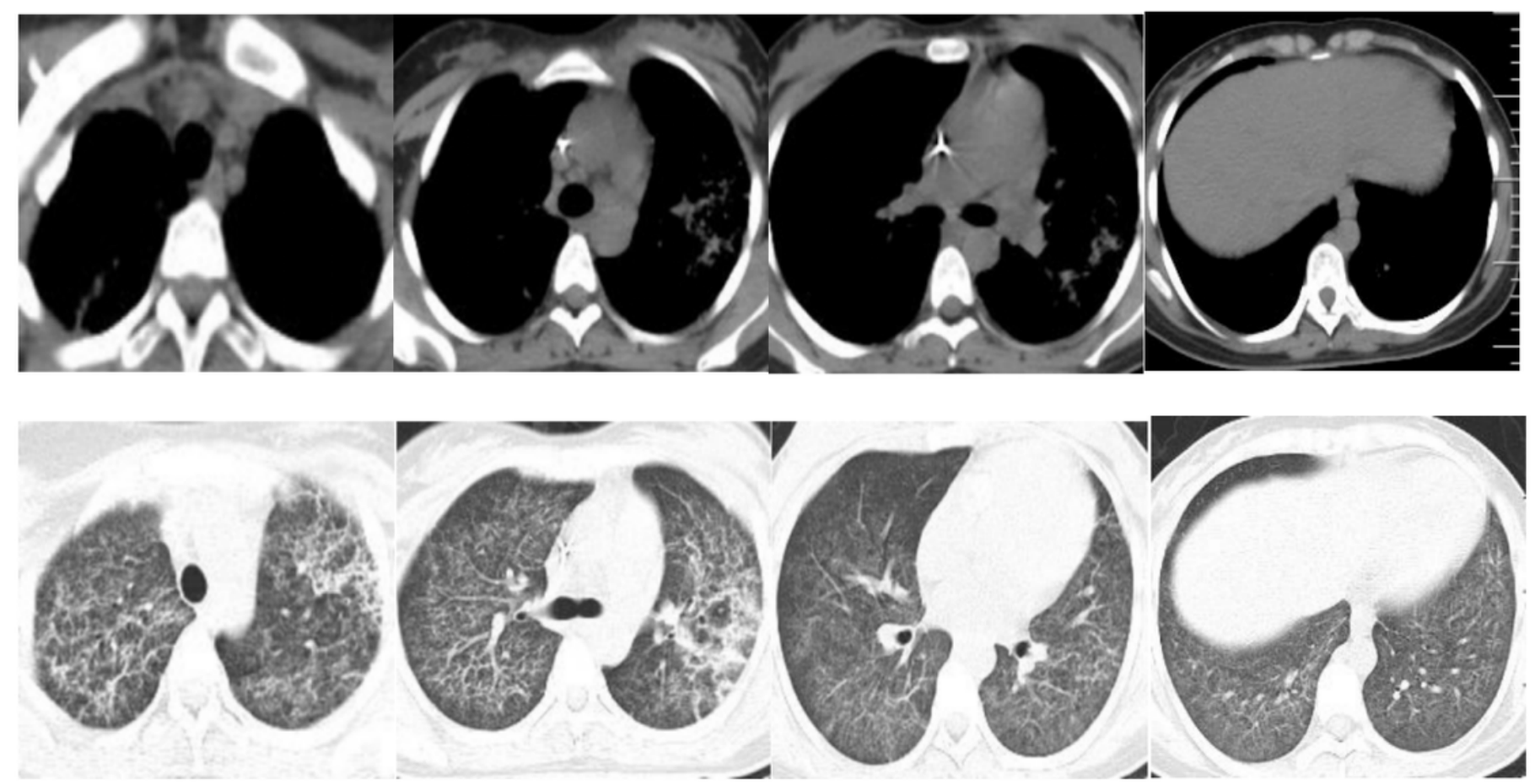

Graph 2

Figure 2 
Chest CT showed significant absorption of both lung lesions(January 27, 2021) 